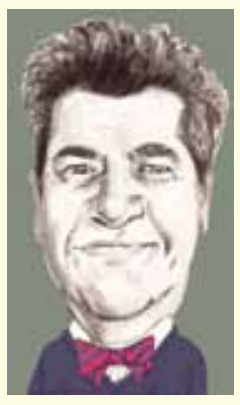

\title{
Protecting students who raise concerns about care delivery
}

\author{
Alan Gasper, emeritus professor at the University of Southampton, discusses revised legislation \\ launched in April 2015, which has been formulated to protect student nurses and midwives from \\ retaliation or victimisation when they raise concerns about things they have witnessed in practice
}

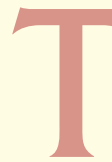

he modified Nursing and Midwifery Council (NMC) code became effective for members of the nursing profession in March 2015. Raising or escalating concerns has been given much greater emphasis in the code, reflecting the concerns about whistleblowing that have been manifest in the wake of a number of NHS scandals where patient safety and care have been compromised (Glasper, 2015).

The NMC code is unequivocal about nurses' and midwives' obligations to raise the concerns they have about service-user safety, and to act on these concerns when they witness them during the course of delivering their duties. The code is very clear that registrants must not obstruct, intimidate or victimise those staff members who are motivated to escalate concerns about patient safety. Making whistleblowing procedures more transparent has been debated within the professional press for a number of years. Interestingly, Mansbach and Bachner (2010) reported the results of a study in which nurses were shown to be much more likely to whistleblow internally rather than externally. Furthermore, as the circle of disclosure widened, this study revealed that nurses were likely to retract their statements of whistleblowing. Similarly, Faugier (2002) discussed how attempts in the recent past by healthcare staff to report on abuse, corruption or malpractice have been largely ignored by senior members of staff. Gallagher (2010) highlighted the dilemma of a former Secretary of State for Health who, after the disclosures (in the Francis Report) of the tragic events that happened at the Mid Staffordshire NHS Foundation Trust, was disappointed that the healthcare staff who worked there during that period had, on the whole, failed to escalate concerns about poor practice by whistleblowing.

Since this watershed in poor care delivery, the Care Quality Commission (CQC) has reorientated its fundamental care standards by introducing the duty of candour. This duty, which was recommended after the unearthing of the events that occurred at Mid Staffordshire, has been introduced to ensure that all staff are required to disclose information about any incident of poor care delivery they believe may have led to patient harm. All healthcare staff must therefore act in an open and transparent way in relation to care and treatment provided to service users. Furthermore, hospitals and other health and social-care facilities must now develop strong procedures for ensuring that staff are open and honest when things go wrong and service users are harmed (CQC, 2014). Since the inquiry into Mid Staffordshire, student nurses in training are taught how to raise concerns about care as part of their programme.

\section{Widening of the Public Interest Disclosure Act to incorporate nursing and midwifery students}

The Public Interest Disclosure Act 1988 was designed to protect people who wish to raise a concern or whistleblow for any detrimental treatment by their employer. The Act and the legislation have now been amended and as of April 2015 offer protection to student nurses and midwives from retaliation or victimisation when they raise concerns. The amended Act now enables student nurses and midwives who are in practice settings as part of their undergraduate education programmes to make protected disclosures to the NMC and other organisations in the same way as registered nurses and midwives currently do. This amendment to the Act was necessary because students working in practice settings, just like professional health workers, may see evidence of poor practice or care delivery, as a result of which service users may be harmed.

The NMC, with its prime mission of public protection, is fully supportive of this change to the legislation and has published advice for nurses and midwives on raising concerns and whistleblowing (NMC, 2015a). This guidance informs nurses how to seek confidential support and advice. Given the emphasis on public protection, the NMC insists that all nurses and midwives have a professional responsibility to ensure that people in their care are fully protected from harm, i.e:

- Physical, psychological or sexual abuse (immediate concerns about abuse should be initially dealt with under local safeguarding procedures)

- Financial or material abuse

- Neglect

a Failure to act or institutional abuse.

In writing this guidance, the NMC recognises the complex dilemmas that nurses experience when escalating concerns about patient safety (Ion et al, 2015). This is because many nurses may not know how to actually raise a concern, or they may have trepidations that they may be perceived by their colleagues as being disloyal, or they may be fearful of employer reprisals.

The loneliness of the healthcare whistleblower is perhaps exemplified in the case of charge nurse Graham Pink who experienced both support and hostility when he raised concerns about staffing levels in an elderly care unit in 1990 (Frais, 2007). It is the stance of the NMC that despite the personal discomfort caused by whistleblowing, each registrant has a prime duty to ensure that the health and wellbeing of people in their care is fully protected. It is important to stress that the early identification of minor concerns can prevent them from becoming more serious and perhaps life-threatening.

\section{Who can registrants and students turn to for help with whistleblowing?}

Professional bodies such as the Royal College of Nursing (RCN) can offer significant levels of support to registrants and students and they have developed guidance to help them decide how best to respond to elements of care they witness, for which they have concerns. This guidance aims to support whistleblowers through the processes involved in reporting concerns when they have reason to believe 
that there might be an act of wrongdoing or misconduct. The RCN (2015) guidance reminds whistleblowers that they are protected by law from harassment, bullying, dismissal and other detrimental actions when they raise concerns in the appropriate way. It is recognised that raising concerns about practice can often seem isolating and intimidating, and professional-body assistance is invaluable to those who whistleblow to protect their patients.

\section{How can registrants and students raise concerns?}

The NMC stipulates that registrants have a professional duty to report any concerns from their workplace that might jeopardise the safety of people in their care. Section 16 of the new NMC code (NMC, 2015b) gives detailed information about raising concerns and-pertinent to students especially-the importance of raising concerns immediately if they are asked to practise beyond their experience and training.

All healthcare workers should act without delay if they believe that there is a risk to patient safety or public protection. Among others, the code asks registrants not to obstruct, intimidate, victimise or in any way hinder a colleague or staff member who wants to raise a concern. The principles of the code now apply to nursing and midwifery students just as they do for registrants, and all students must inform their mentor, personal tutor or lecturer immediately if they believe that that any patient may be at risk of harm. Students must seek help immediately from an appropriately qualified professional if someone they are providing care to has suffered harm for any reason.

The NMC recognises that students may find all of this overwhelming and they recommend that students discuss their concerns with their university tutor or lecturer, or mentor. The NMC code also reminds students that they must respect a service user's right to confidentiality.

Only in very exceptional circumstances may a student disclose information about a service user without their permission-when they genuinely believe that they may be at risk of harm and are therefore acting in the service user's best interests.

\section{Guidance for students on raising concerns relating to practice}

In the case of students wishing to raise concerns, it is therefore the university tutor, lecturer, mentor or practice education facilitator who will provide frontline advice. The NMC expects all universities offering undergraduate preregistration training to have developed a 'raising concerns' protocol or algorithm in consultation with their practice partners. This algorithm or flowchart should be prominent in clinical areas and is often part of the assessment of practice documentation given to students while they are in practice. Typical university protocols for helping students understand the correct procedures for raising a practicerelated concern will explain a number of stages that students must go through.

The first stage is deciding whether immediate or prompt action is required. Here, the university will instruct the student to report their concerns immediately to the person in charge or to the mentor, where they should provide them with details of what was witnessed and who was involved. Often issues can be resolved at this stage.

If it progresses to the next stage, the mentor or person in charge of the clinical area should use the local process for raising concerns by following the protocol. Hence, the senior manager may visit the area to make an initial assessment. The mentor, or student, would then normally contact the academic tutor. This process may result in an investigation by the practice area, and the university may appoint someone to gather further information, possibly involving obtaining the perspectives of others involved. Students may at this stage be asked to write a statement. It is important that students seek advice about this from their personal tutors before preparing this.

If concerns are not fully resolved and the student remains worried that patient risk still remains, the student will be helped by the university to escalate this concern further, usually to the director of nursing. If these concerns are not adequately addressed, the university may refer these concerns to a relevant regulatory body, such as the NMC.

\section{Raising concerns to a regulatory body such as the CQC}

Although this new protection offered to student whistleblowers is given by the Public Interest Disclosure Act, the NMC recommends that before contacting any regulatory bodies or other external agencies, students take due diligence of any university protocol and take advice from their personal tutors. Students should only raise a concern externally when all other procedures available to them have been followed and exhausted. It is important to stress that raising a concern externally without clear evidence that appropriate protocols have been followed might jeopardise the legitimacy of that action.

BJN

Care Quality Commission (2014) Publishing of the new fundamental standards. http://tinyurl.com/psrkyrq (accessed 5 May 2015)

Faugier J (2002) Valuing ‘voices from below'. J Nurs Manag 10(6): 315-20

Frais (2007) Why whistleblowing 'heroes' such as Pink undermine the practice of whistleblowing. $B M J$ online http://tinyurl.com/o8kpy3t (accessed 18 May 2015)

Gallagher A (2010) Whistleblowing: what influences nurses' decisions on whether to report poor practice? Nurs Times 106(4): 22-5

Glasper A (2015) Can the new NMC Code improve standards of care delivery? Br J Nurs 24(4): 186-7

Ion R, Smith K, Nimmo S, Rice AM, McMillan L (2015) Factors influencing student nurse decisions to report poor practice witnessed while on placement. Nurse Educ Today: in press. doi: http://dx.doi.org/10.1016/j. nedt.2015.02.006

Mansbach A, Bachner YG (2010) Internal or external whistleblowing: nurses' willingness to report wrongdoing. Nurs Ethics 17(4): 483-90. doi: 10.1177/0969733010364898

Nursing and Midwifery Council (2015a) Raising Concerns: Guidance for Nurses and Midwives. Revised. http:// tinyurl.com/o2khlh9 (accessed 18 May 2015)

Nursing and Midwifery Council (2015b) The Code: Professional Standards of Practice and Behaviour for Nurses and Midwives. http://www.nmc.org.uk/standards/ code/ (accessed 18 May 2015)

Royal College of Nursing (2015) Raising concerns: a guide for RCN members. http://tinyurl.com/pmpazdt (accessed 18 May 2015)

\section{KEY POINTS}

- The Public Interest Disclosure Act now protects student nurses and midwives from retaliation or victimisation when they raise concerns about things they have witnessed in practice

- The NMC code is unequivocal about nurses' and midwives' obligations to raise the concerns they have about service user safety

- The loneliness of the healthcare whistleblower may make nurses fearful of raising concerns

- Good practice suggests that all universities offering undergraduate preregistration training should develop a 'raising concerns' protocol or algorithm in consultation with their practice partners

Students should only raise a concern externally when all other procedures available to them have been followed and exhausted 